\title{
BMJ Open Hypertension treatment practices and its determinants among ambulatory patients: retrospective cohort study in Ethiopia
}

\author{
Derbew Fikadu Berhe, ${ }^{1,2}$ Katja Taxis, ${ }^{3}$ Flora M Haaijer-Ruskamp, ${ }^{1}$ \\ Afework Mulugeta, ${ }^{4}$ Yewondwossen Tadesse Mengistu, ${ }^{5}$ Peter G M Mol ${ }^{1}$
}

To cite: Berhe DF, Taxis $\mathrm{K}$, Haaijer-Ruskamp FM, et al. Hypertension treatment practices and its determinants among ambulatory patients: retrospective cohort study in Ethiopia. BMJ Open 2017;7:e015743. doi:10.1136/ bmjopen-2016-015743

- Prepublication history and additional material are available. To view these files, please visit the journal online (http://dx.doi. org/10.1136/bmjopen-2016015743).

Received 28 December 2016 Revised 9 June 2017 Accepted 28 June 2017

\section{CrossMark}

${ }^{1}$ Department of Clinical Pharmacy and Pharmacology, University of Groningen, University Medical Center Groningen, Groningen, The Netherlands

${ }^{2}$ Department of Pharmacy, Mekelle University, Mekelle, Tigray, Ethiopia

${ }^{3}$ Department of Pharmacy, Unit Pharmacotherapy, Epidemiology and Economics, University of Groningen, Groningen, Netherlands

${ }^{4}$ School of Public Health, College of Health Sciences, Mekelle University, Mekelle, Tigray, Ethiopia

${ }^{5}$ Department of Internal Medicine, School of Medicine, College of Health Sciences, Addis Ababa University, Addis Ababa, Ethiopia

Correspondence to Dr Peter G M Mol; p.g.m.mol@umcg.nl

\section{ABSTRACT}

Objectives We examined determinants of achieving blood pressure control in patients with hypertension and of treatment intensification in patients with uncontrolled blood pressure (BP).

Design A retrospective cohort study in six public hospitals, Ethiopia.

Participants Adult ambulatory patients with hypertension and with at least one previously prescribed antihypertensive medication in the study hospital.

Outcome Controlled BP ( $<140 / 90 \mathrm{~mm} \mathrm{Hg})$ and treatment intensification of patients with uncontrolled BP. Results The study population comprised 897 patients. Their mean age was 57 (SD 14) years, 63\% were females, and $35 \%$ had one or more cardiometabolic comorbidities mainly diabetes mellitus. BP was controlled in $37 \%$ of patients. Treatment was intensified for $23 \%$ patients with uncontrolled BP. In multivariable (logistic regression) analysis, determinants positively associated with controlled BP were treatment at general hospitals (OR 1.89, 95\% Cl 1.26 to 2.83) compared with specialised hospitals and longer treatment duration (OR 1.04, 95\% Cl 1.01 to 1.06 ). Negatively associated determinants were previously uncontrolled $\mathrm{BP}(\mathrm{OR} 0.30,95 \% \mathrm{Cl} 0.21$ to 0.43 ), treatment regimens with diuretics (OR $0.68,95 \%$ $\mathrm{Cl} 0.50$ to 0.94 ) and age (OR $0.99,95 \% \mathrm{Cl} 0.98$ to 1.00 ). The only significant-positive-determinant for treatment intensification was duration of therapy (OR 1.05, 95\% Cl 1.02 to 1.09 ).

Conclusions The level of controlled BP and treatment intensification practice in this study was low. The findings suggest the need for in-depth understanding and interventions of the identified determinants such as uncontrolled BP on consecutive visits, older age and type of hospital.

\section{BACKGROUND}

Hypertension is a major risk factor for cardiovascular diseases, and it is the leading cause of death and disability globally. ${ }^{1}$ The WHO recently reported that $80 \%$ of deaths due to cardiovascular disease occur in low and middle-income countries, with the highest death rate reported in African countries. The

\section{Strengths and limitations of this study}

- This is the first study that gives insight into determinants of hypertension treatment practice (level of blood pressure (BP) control and treatment intensification) in a diverse population treated in public hospitals in Ethiopia.

- We analysed BP measurements as recorded in patient medical records, which reflect actual clinical practice, but may be subject to recording and measurement error.

- The finding of this study may not be generalisable to other settings such as private practice or primary healthcare centres in Ethiopia.

report also indicated that prevalence of hypertension in adults was higher in Africa (46\%) than for instance in the USA $(35 \%) .{ }^{1}$ Hypertension is also more prevalent among people from Africa living in the Western world than among whites. ${ }^{2}$ The population of African ancestry is characterised by high vascular contractility, extreme salt sensitivity and low renin release. ${ }^{3-5}$ Hence, overactivation of the sympathetic nervous system and renin-angiotensin-aldosterone system is more prevalent in this population, making them more vulnerable to high blood pressure (BP). In addition, changes in environmental factors such as economic development, urbanisation and lifestyle have resulted in an epidemiological transition from infectious to non-communicable disease such as hypertension in the African region. ${ }^{6}$

Large clinical outcome studies have repeatedly shown that treating hypertension using evidence-based antihypertensive treatment and/or adjusting lifestyle improves cardiovascular outcomes. ${ }^{7}$ However, achieving target BP levels remains a challenge in clinical practice. The majority of studies in Africa have shown that less than a third of patients 
achieve treatment goals. ${ }^{8}$ Generally, four main factors have been identified that influence achieving controlled BP. First, there are factors intrinsic to the nature of the disease; in most cases hypertension is initially asymptomatic and this delays early prevention, diagnosis and treatment. ${ }^{9}$ Second, poor treatment response may be due to patient-related factors such as age, gender, race, awareness and compliance to medication. ${ }^{410}$ Third, there are healthcare system-related factors such as lack of effective hypertension prevention and treatment programmes and access to medications. Fourth, prescriber behaviour, competences and large patient:prescriber ratio affect hypertension prevention and treatment outcomes. The majority of these factors have been extensively studied in western societies; however, little is known of their impact on BP control in developing nations. Some of these factors may be unique to, or more pronounced in the African setting, including low societal awareness, priority to fight infectious diseases and human resource limitations, in particular the number of available healthcare professionals. ${ }^{611}$

Prevention and treatment strategies have been shown to be effective in optimising $\mathrm{BP}$ control in the western world. $^{12}$ Such programmes may be relevant for the African setting. To guide targeted interventions, studies identifying factors contributing to poor BP control in the African setting are urgently needed. Studies on hypertension conducted to date in Ethiopia, the second most populous country (approximately 100 million) in Africa, have focused on determining prevalence of the disease. ${ }^{13-15}$ Prevalence is relatively low $(10 \%-30 \%),{ }^{13-15}$ but further data on hypertension treatment practices are lacking. ${ }^{1617}$ Therefore, we aimed to assess the proportion of patients treated for hypertension who had controlled BP and identify determinants for achieving BP control in an Ethiopian setting. Additionally, we aimed to study whether treatment was intensified in those patients with uncontrolled BP and identify the determinants for treatment intensification.

\section{METHODS}

\section{Study design and setting}

This retrospective cohort study was conducted in outpatient clinics of six public hospitals in Addis Ababa (capital city) and Tigray regional state, Ethiopia. We included two specialised hospitals (Addis Ababa) and four general hospitals, three from Tigray and one from Addis Ababa. Specialised (tertiary) hospitals are at the top tier of the Ethiopian public healthcare system and serve approximately 5 million people. The general (secondary) hospitals are estimated to serve $1-1.5$ million people. Furthermore, patients including those with hypertension are usually treated first at a primary healthcare centre. ${ }^{18}$

\section{Study population}

Participants were approached while waiting for their appointment in the waiting area of hypertension outpatient clinics, where known patients with hypertension come for regular follow-up visits. Participants were recruited consecutively after giving consent. Patients with hypertension aged 18 years or older were included, if they had at least one previous antihypertensive medication prescription in the same hospital and gave informed consent. Patients were identified based on self-reported hypertension or based on a mark on their pocket-size appointment card as being hypertensive. We verified in each clinic log book (if available) and from individual patient medical records if patients met the inclusion criteria as they had indicated during the interviews.

Routine practice in the study hospitals is that nurses measure the patient's BP and assign the patient to a physician. The physician then performs a consultation, confirms the hypertension diagnosis, if necessary performs further examinations including rechecking BP and renews or amends prescribed medication. Patients then collect their medicine from pharmacy outlets at the same hospital or from private or community pharmacies.

\section{Data collection}

Included patients were interviewed in the waiting area before they were seen by the physician. Data collected via interview included sociodemographics, medication adherence and duration of antihypertensive treatment with medication. The sociodemographics variables were age, sex, educational and marital status, alcohol use and smoking habits. Clinical information retrieved from medical records were BP measurements, medication prescriptions and comorbid illnesses, and information was retrieved for the current visit and the previous (prior) visit. Data were collected by professional nurses or pharmacists who were trained in using a dedicated case report form. Data were collected between February and August 2015.

\section{Variables}

\section{Outcome measures}

We defined two outcome measures. First, for BP control we used the 'standard' definition of controlled BP, that is, systolic BP/diastolic BP below $140 / 90 \mathrm{~mm} \mathrm{Hg}$ at the current visit. ${ }^{12}$ Second, we defined treatment intensification as an increase in dose of an antihypertensive drug and/or addition of one or more antihypertensive $\operatorname{drug}(\mathrm{s})$. Treatment intensification was calculated for those patients who had a complete medication history (including dose and administration frequency) at both current and prior visits and whose BP was not controlled at the current visit. A switch in drug class was not considered as treatment intensification.

\section{Explanatory variables}

For determinants of BP control or treatment intensification, we included sociodemographic variables (age in year, gender, smoking history, alcohol use, marital status and educational status), hospital type (general vs specialised), cardiometabolic comorbid illnesses (diabetes mellitus (DM), dyslipidaemia, kidney disease, heart failure/ myocardial infarction), uncontrolled BP $(\geq 140 / 90 \mathrm{~mm}$ 
$\mathrm{Hg})$ at the prior visit, duration of antihypertensive treatment in years, treatment adherence with the eight-point Morisky Medication Adherence Scale (MMAS-8; $\geq 7$ : yes/ no), visit schedule in month and antihypertensive medications prescribed at the prior visit. For alcohol use and smoking habit, participants were asked if they were active smokers or consume alcohol up to our survey date, that is, smoking history (Yes: current smokers; No: never smoke or ex-smoker), alcohol use (Yes: regularly or sometimes; No: never consume alcohol). The visit schedule was calculated by taking the difference in days between the current and prior visits divided by 30 , that is, indicating the length of time (duration) between the two follow-up visit expressed in months.

Antihypertensive medication adherence was measured with MMAS-8, which has been previously validated for patients with hypertension. ${ }^{19} 20$ The items of the scale are grouped into three aspects. The first aspect is about sometimes forgetting or intentionally not taking prescribed medication (item 1), and more specifically in the past 2 weeks (item 2), or under special circumstances during travel/leaving home (item 4) and finally asking if medication was taken yesterday (item 5). The second aspect is about intentionally stopping or cutting back medication because of feeling worse (item 3) or because of a feeling that $\mathrm{BP}$ is under control (item 6). The last aspect relates to convenience (item 7) or inconvenience frequency of difficult times to take medication (item 8). The scale was translated into two Ethiopian languages (Amharic and Tigrigna) according to the method described by Beaton et $a l .{ }^{21} \mathrm{~A}$ total score of seven or more (maximum eight) was considered to be adherent to antihypertensive medication; that is, MMAS $\geq 7 .{ }^{19}$ For a sensitivity analyses, we used a lower level of adherence with a cut-off of MMAS $\geq 6$.

\section{Sample size}

Achieved sample size was based on an estimated 30\% prevalence of controlled BP among treated patients with hypertension in Ethiopia ${ }^{815}$ and a single proportion sample size calculation formula. The total sample size was 984 (164 per hospital) with 0.80 power, $95 \%$ CI, 3\% margin of error and an estimated $10 \%$ none response rate or incomplete/irretrievable patient records.

\section{Statistical analyses}

Descriptive statistics were used to summarise sociodemographic, disease characteristics of the study population and nature and frequency of antihypertensive medications used. Multivariable logistic regression analyses were applied to investigate determinants for achieving target $\mathrm{BP}$ at current visit and determinants for treatment intensification. Statistical significance was considered at $p$ value $<0.05$. Potential determinants with $\mathrm{p}<0.2$ in bivariable analyses were included into the multivariable logistic model. Microsoft Access V.10 and SPSS V.22.0 statistical software was used for data entry and analyses, respectively.

\section{Sensitivity analyses}

We performed five sensitivity analyses. First, tighter BP targets at the current visit (BP $<130 / 80 \mathrm{~mm} \mathrm{Hg}$ ) were applied for those patients with diabetes mellitus (DM) and/or renal disease. Standard BP target $(\mathrm{BP}<140 / 90 \mathrm{~mm}$ $\mathrm{Hg}$ ) was used for all others participants. Second, we performed a sensitivity analysis for the main outcome measure (controlled BP <140/90) using a different cut-off for adherence (MMAS $\geq 6$ ). Third (for controlled BP) and fourth (for treatment intensification) sensitivity analysis were similar to the main analysis with three modified determinants. Graded hypertension (prior BP) was performed according to the stages defined by the Ethiopian standard treatment guideline for hypertension: normal BP (systolic BP $<120$ and $\mathrm{DBP}<80 \mathrm{~mm} \mathrm{Hg}$ ), prehypertensive stage (systolic BP 120-139 or diastolic BP 80-89 $\mathrm{mm} \mathrm{Hg}$ ), stage I hypertension (systolic BP 140-159 or diastolic BP 90-99 $\mathrm{mm} \mathrm{Hg}$ ) and stage-II hypertension (systolic BP $\geq 160$ or diastolic $\mathrm{BP} \geq 100 \mathrm{~mm} \mathrm{Hg}$ ). ${ }^{22}$ These analyses also included the number of cardiometabolic comorbid illnesses as a proxy measure for more severely ill patients and age categorised into five groups. Patients with higher hypertension stages and multiple comorbid illness were hypothesised to be more difficult to treat. A fifth sensitivity analysis was performed in patients who had been on medication for at least 6 months, assuming that these patients were no longer in the initial careful uptitration phase.

\section{Ethics approval}

This study was approved by Ethiopian Health Research Ethical Review Committees of (1) the College of Health Sciences, Mekelle University, (2) St Paul's Hospital Millennium Medical College, and (3) the Department of Internal Medicine, School of Medicine, College of Health Sciences, Addis Ababa University. All individual participants included in this study consented to participation.

\section{RESULTS}

We were able to approach 968 patients at six public hospitals in Ethiopia. Seventy-one patients were excluded from our analyses: eight refused to participate, six were not hypertensive and 57 patients had no retrievable records or incomplete records (missing BP at current visit). This resulted in a study population of 897 patients (figure 1). The majority of included patients $(93 \%)$ reported to have come for their regular hypertension follow-up visit. The remaining $7 \%$ had (perceived) symptoms, uncontrolled hypertension or adverse events. The mean (SD) patient age was 57 (14) years, $63 \%$ of patients were female, most patients $(65 \%)$ were married and $64 \%$ had no formal education or only attended primary school. Almost all (94\%) had never smoked, and nearly half (43\%) consumed alcohol regularly or occasionally (table 1 ). At the current visit, $35 \%$ study participants had at least one recorded comorbid illness, predominantly DM (table 1 ).

Thirty-seven per cent $(n=335)$ of the participants had controlled BP at the current visit (table 1). Applying the stricter BP target for patients with DM and/or renal 


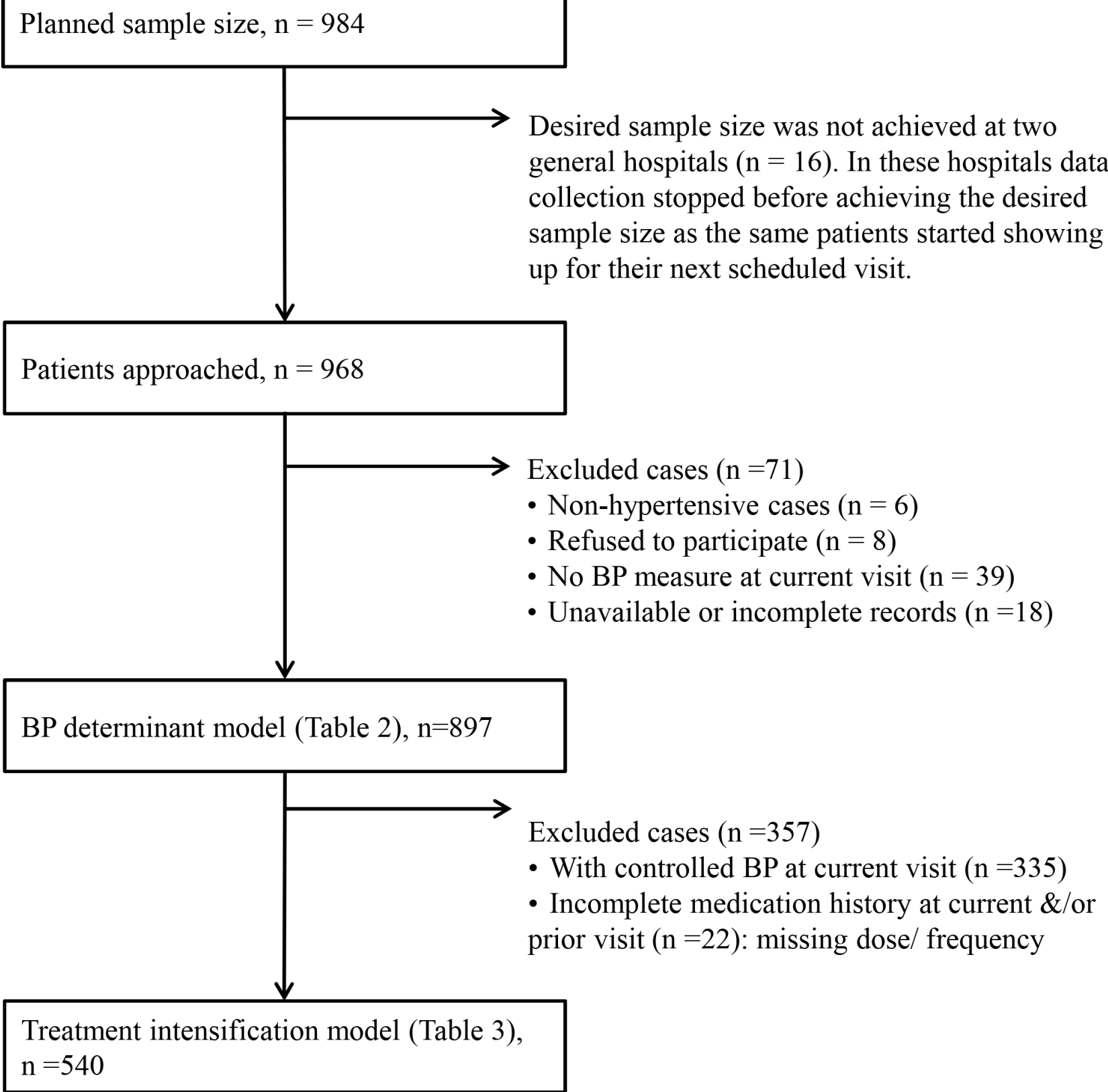

Figure 1 Flow chart for case inclusion for analysis.

disease $(\mathrm{BP}<130 / 80 \mathrm{~mm} \mathrm{Hg}$ ), the proportion of patients with controlled BP dropped to $27 \%(n=231)$.

Only $23 \%$ of 540 patients with uncontrolled BP and complete medication history had their treatment intensified (table 1). For $22(4 \%)$ of 562 patients with uncontrolled BP at the current visit, the medication history was not complete. Either the dose and/or administration frequency were missing. The antihypertensive medication adherence rate (MMAS $\geq 7$ ) was $40 \%$ (table 1) and $57 \%$ for the lower cut-off, MMAS $\geq 6$ (see online supplementary table 1).

Overall, ACE inhibitors were the most commonly prescribed group of drugs $(n=503)$, followed by diuretics $(\mathrm{n}=498)$ (table 1). Medication use was quite similar on both visits. At the current visit, $62 \%$ of included patients were prescribed a multidrug treatment regimen and $45 \%$ patients took two antihypertensive agents (see online supplementary table 2). Most (38\%) of the 
Table 1 Characteristics of ambulatory patients with hypertension at two visits, Ethiopia

\section{Characteristics}

Current visit

Prior visit

Demographics

$\begin{array}{lc}\text { Age (mean, SD), year } & 57(14) \\ \text { Female (n, \%) } & 551(63) \\ \text { Smoking (current smoker) (n, \%) } & 57(6) \\ \text { Alcohol use (regularly or sometimes) (n, \%) } & 378(43) \\ \text { Married (n, \%) } & 567(65) \\ \text { Education (n, \%) } & 170(20) \\ \quad \text { University/college education } & 141(16) \\ \quad \text { Secondary education } & 557(64)\end{array}$

Setting

Specialised hospitals: both from Addis Ababa (n, \%)

Tikur Anbessa Hospital

$139(16)$

St Paul's Hospital

$153(17)$

General hospitals: all from Tigray, except Yekatit 12 from Addis Ababa (n, \%)

St Mary Axum Hospital

$139(16)$

Mekelle Hospital

$152(17)$

Lemlem Karl Maychew Hospital

155 (17)

Yekatit 12 Hospital

$159(18)$

Disease characteristics

BP

Systolic BP (Mean, SD)

$139(21) \quad 144(22)$

Diastolic BP (Mean, SD)

84 (11)

85 (13)

Controlled BP (<140/90 mm Hg) (n, \%)

$335(37)$

$231(27)$

Controlled BP $(<130 / 80 \mathrm{~mm} \mathrm{Hg}$ with DM and/or kidney diseases, *otherwise

$268(30)$

$202(24)$

$<140 / 90 \mathrm{~mm} \mathrm{Hg})(\mathrm{n}, \%)$

Cardiometabolic comorbid illnesses (n, \%)

$\begin{array}{lcc}\text { Diabetes mellitus } & 227(25) & 198(22) \\ \text { Dyslipidaemia } & 57(6) & 45(5) \\ \text { Renal diseases } & 25(3) & 23(3) \\ \text { Heart failure / myocardial infarction } & 72(8) & 60(7)\end{array}$

Antihypertensive treatment characteristics

Drug class ( $\mathrm{n}, \%)$

ACE inhibitors

Enalapril

$503(56)$

$494(55)$

$499(56)$

$492(55)$

Lisinopril or captopril

$4(0.4)$

$2(0.2)$

Beta blockers

Atenolol

$167(19)$

166 (19)

$148(17)$

147(16)

Propranolol, metoprolol or carvedilol

Calcium channel blockers

Nifedipine

$19(2)$

$19(2)$

$449(50)$

$439(49)$

Amlodipine or felodipine

Diuretics†

$381(43)$

$389(43)$

$68(8)$

$50(6)$

Hydrochlorothiazide

498 (56)

486 (54)

Furosemide

428 (48)

421 (47)

$76(9)$

71 (8)

Continued 


\begin{tabular}{|c|c|c|}
\hline Characteristics & Current visit & Prior visit \\
\hline Spironolactone & $72(8)$ & $66(7)$ \\
\hline Others (methyldopa, nitrates or losartan) & $19(2)$ & $13(1)$ \\
\hline Duration of therapy years (median, IQR) & $4(7)$ & \\
\hline Visit schedule in months (mean, SD) & $2.3(2.0)$ & \\
\hline Adherence (MMAS $\geq 7)(n, \%)$ & $355(40)$ & \\
\hline \multicolumn{3}{|l|}{ Therapy (n, \%) } \\
\hline Monotherapy & $343(38)$ & $363(41)$ \\
\hline Multidrug therapy & $550(62)$ & $521(59)$ \\
\hline $\begin{array}{l}\text { Treatment intensified in patients with uncontrolled BP at current visit }(n=540) \neq \\
(n, \%)\end{array}$ & $123(23)$ & \\
\hline
\end{tabular}

Mono/multidrug therapy is limited to antihypertensive medications.

${ }^{*}$ Otherwise: patients with hypertension without DM or kidney disease.

†Some patients had more than one type of diuretics.

‡For 22 of 562 patients with uncontrolled BP at the current visit, the medication history was not complete. Treatment intensification could thus only be calculated for 540 patients.

BP, blood pressure; DM, diabetes mellitus; MMAS, Morisky Medication Adherence Scale.

343 patients on monotherapy were prescribed diuretics $(\mathrm{n}=127)$.

\section{Determinants of BP control}

$\mathrm{BP}<140 / 90 \mathrm{~mm} \mathrm{Hg}$ (primary analysis)

According to our multivariable logistic regression model (table 2), factors significantly associated with achieving target $\mathrm{BP}$ at the current visit were age (OR $0.99,95 \%$ CI 0.98 to 1.00 ), follow-up at general hospitals (OR 1.89, $95 \%$ CI 1.26 to 2.83), inadequately controlled BP at prior visit (OR $0.30,95 \%$ CI 0.21 to 0.43 ), longer treatment duration per year (OR 1.04, 95\% CI 1.01 to 1.06 ) and prescribed diuretics (OR $0.68,95 \%$ CI 0.50 to 0.94 ).

\section{Determinants of treatment intensification}

The only statistically significant determinant for treatment intensification in the multivariable analyses was longer treatment duration (OR $1.05,95 \%$ CI 1.02 to 1.09 ) (table 3).

\section{Sensitivity (secondary) analyses}

In our first sensitivity analyses, using $\mathrm{BP}<130 / 80 \mathrm{~mm} \mathrm{Hg}$ for patients with DM and/or renal disease, and for all other patients $<140 / 90 \mathrm{~mm} \mathrm{Hg}$ as cut-offs, uncontrolled BP at the prior visit had a negative effect on achieving target $\mathrm{BP}$ at the current visit (OR $0.25,95 \%$ CI 0.17 to 0.37 ). Medications prescribed during the prior visit, except diuretics (OR $0.60,95 \%$ CI 0.40 to 0.90 ), were not significantly associated with achieving controlled BP at the current visit. Unlike the primary analyses, age, treatment duration and hospital type did not show statistically significant effects on current visit BP status (see online supplementary table 3 ).

In the sensitivity analyses for BP control (see online supplementary table 4) and treatment intensification (see online supplementary table 5), the results were mostly similar with the main analysis (tables 2 and 3 , respectively). As expected, more severe hypertension stage was associated with more difficulty to achieve target BP: stage II hypertension (OR $0.17,95 \%$ CI 0.09 to 0.35 ) and stage I hypertension (OR $0.34,95 \%$ CI 0.17 to 0.67 ). However, the number of comorbid illnesses was not a significant determinant of achieving target BP. In case of age, older age groups were less likely to achieve target $\mathrm{BP}$ than younger age groups ( $<35$ years) : 55-64 years old (OR $0.41,95 \%$ CI 0.20 to 0.83 ) and $\geq 65$ years old (OR 0.46 , 95 CI 0.22 to 0.93$)$. Supplementary analysis for treatment intensification (see online supplementary table 5) gave similar results with main analysis on table 3 , where only duration of therapy was a significant determinant (OR $1.05,95 \%$ CI 1.02 to 1.08 ) for more treatment intensification. The majority (94\%) of participants had been on medication for at least for 6 months. Exclusion of the $6 \%$ of patients who had recently started therapy $(<6$ months ago) in the sensitivity analysis (see online supplementary table 6) did not change our findings reported in table 2. The proportion of patients with controlled BP (39\%) remained similar as well. Duration of therapy remained a significant determinant for achieving target BP and for intensifying treatment.

\section{DISCUSSION}

In this study, nearly two-thirds of patients on antihypertensive medication had uncontrolled BP. Drugs were prescribed from four antihypertensive drug classes, ACE inhibitors, diuretics, calcium channel blockers and beta blockers. Generally, a single-specific agent (over $90 \%$ ) was prescribed within a class, enalapril, hydrochlorothiazide, nifedipine and atenolol, respectively. Age of patients, uncontrolled $\mathrm{BP}$ at the prior visit and a treatment regimen containing diuretics contributed to poorer BP control. Follow-up in a general hospital compared with a specialised hospital and longer treatment duration were 
Table 2 Determinants of achieving target BP $(\mathrm{BP}<140 / 90)$ at current visit in ambulatory patients with hypertension

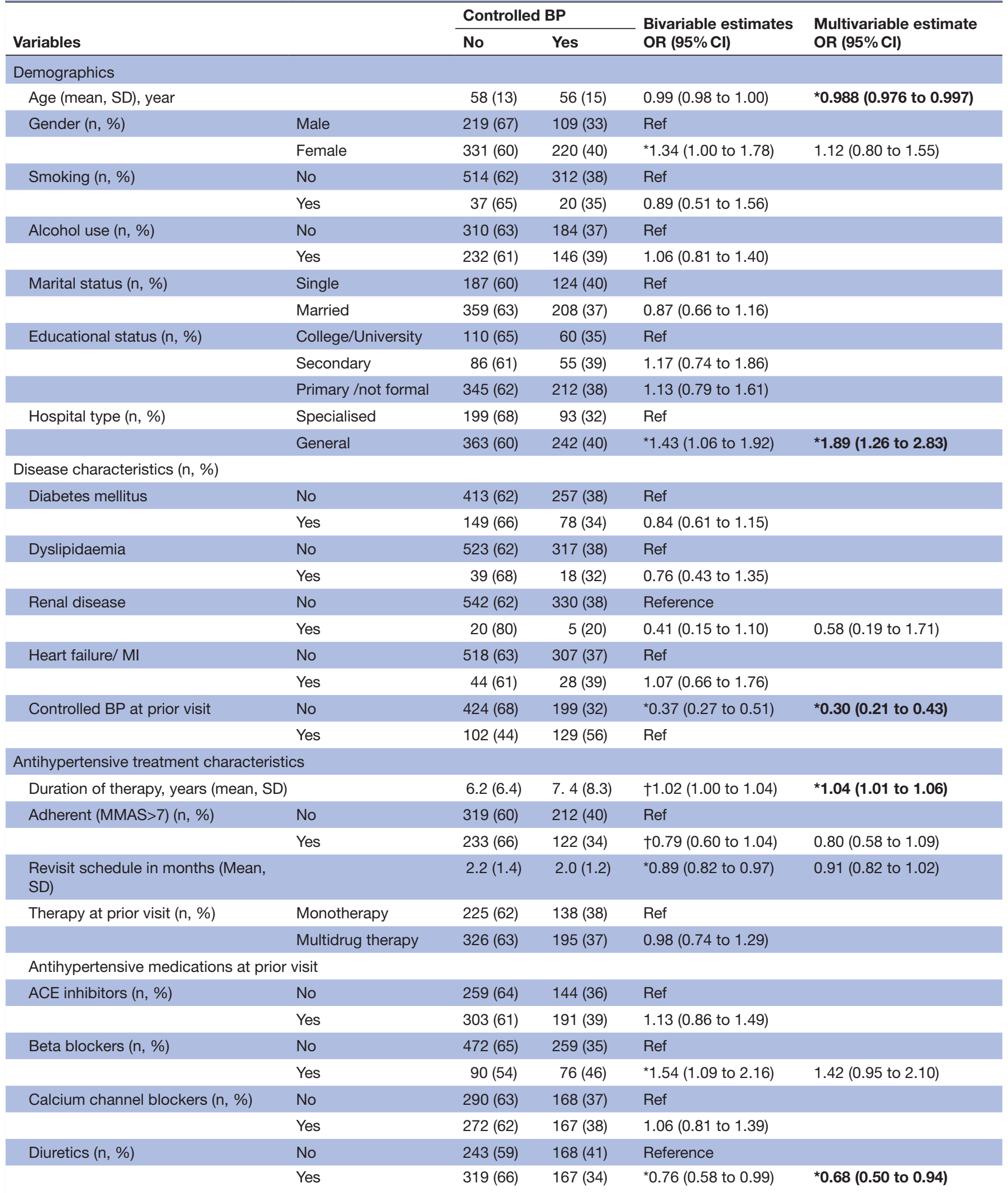

Only patients with available data were included in the analyses, therefore numbers may sometimes differ from table 1 . Percentages are calculated per row. Statistically significant values: ${ }^{*} p<0.05$. Variables with ${ }^{*} p<0.05$ or $\uparrow p<0.20$ in the bivariable model were included in the multivariable model. Variables with a $p<0.05$ in the multivariable model are indicated with * and set in bold typeface. Mono/Multidrug therapy was for antihypertensive medications.

BP, blood pressure; MMAS, Morisky Medication Adherence Scale; MI, myocardial infarction; Ref, reference. 
Table 3 Treatment intensification determinants for ambulatory patients with hypertension with uncontrolled BP at current visit

\begin{tabular}{|c|c|c|c|c|c|}
\hline \multirow{2}{*}{ Variables } & & \multicolumn{2}{|c|}{$\begin{array}{l}\text { Treatment } \\
\text { intensified }\end{array}$} & \multirow{2}{*}{$\begin{array}{l}\text { Bivariable } \\
\text { estimates OR } \\
(95 \% \mathrm{Cl})\end{array}$} & \multirow{2}{*}{$\begin{array}{l}\text { Multivariable } \\
\text { estimate } \\
\text { OR }(95 \% \mathrm{Cl})\end{array}$} \\
\hline & & No & Yes & & \\
\hline \multicolumn{6}{|l|}{ Demographics } \\
\hline Age (mean, SD), Year & & $57(13)$ & $60(13)$ & & $1.02(1.00$ to 1.04$)$ \\
\hline \multirow[t]{2}{*}{ Gender (n, \%) } & Male & $167(80)$ & $41(20)$ & Ref & \\
\hline & Female & $241(75)$ & $80(25)$ & $\dagger 1.35$ (0.88 to 2.07$)$ & $1.47(0.91$ to 2.37$)$ \\
\hline \multirow[t]{2}{*}{ Smoking (n, \%) } & No & $383(77)$ & $113(23)$ & Ref & \\
\hline & Yes & $27(79)$ & $7(21)$ & 0.88 (0.37 to 2.07$)$ & \\
\hline \multirow[t]{2}{*}{ Alcohol use $(n, \%)$} & No & $225(76)$ & $73(25)$ & Ref & \\
\hline & Yes & $180(80)$ & $44(20)$ & 0.75 (0.49 to 1.15$)$ & \\
\hline \multirow[t]{2}{*}{ Marital status (n, \%) } & Single & $138(77)$ & $42(23)$ & Ref & \\
\hline & Married & $269(78)$ & $76(22)$ & 0.93 (0.60 to 1.43$)$ & \\
\hline \multirow[t]{3}{*}{ Educational status ( $n, \%)$} & College/University & $82(78)$ & $23(22)$ & Ref & \\
\hline & Secondary & $58(70)$ & $25(30)$ & 1.54 (0.80 to 2.97$)$ & \\
\hline & $\begin{array}{l}\text { Primary /not } \\
\text { formal education }\end{array}$ & $262(79)$ & $70(21)$ & 0.95 (0.56 to 1.62$)$ & \\
\hline \multirow[t]{2}{*}{ Hospital type (n, \%) } & Specialised & $137(73)$ & $51(27)$ & Ref & \\
\hline & General & $280(80)$ & $72(21)$ & †0.69 (0.46 to 1.04$)$ & 0.83 (0.51 to 1.37$)$ \\
\hline \multicolumn{6}{|l|}{ Disease characteristics (n, \%) } \\
\hline \multirow[t]{2}{*}{ Diabetes mellitus at current visit } & No & $311(79)$ & $84(21)$ & Ref & \\
\hline & Yes & $106(73)$ & $39(27)$ & $\dagger 1.36$ (0.88 to 2.11$)$ & 1.10 (0.67 to 1.81$)$ \\
\hline \multirow[t]{2}{*}{ Dyslipidaemia at current visit } & No & $388(77)$ & $113(23)$ & Reference & \\
\hline & Yes & $29(74)$ & $10(26)$ & 1.19 (0.56 to 2.50$)$ & \\
\hline \multirow[t]{2}{*}{ Renal disease at current visit } & No & $403(77)$ & $118(23)$ & Ref & \\
\hline & Yes & $14(74)$ & $5(26)$ & 1.22 (0.43 to 3.45$)$ & \\
\hline \multirow[t]{2}{*}{ Heart Failure/Ml at current visit } & No & $384(77)$ & $112(23)$ & Ref & \\
\hline & Yes & $33(75)$ & $11(25)$ & 1.14 (0.56 to 2.33$)$ & \\
\hline \multirow[t]{2}{*}{ Controlled BP at prior visit } & No & $312(77)$ & $96(24)$ & $\dagger 1.50$ (0.85 to 2.66$)$ & 1.38 (0.76 to 2.50$)$ \\
\hline & Yes & $83(83)$ & $17(17)$ & Ref & \\
\hline \multicolumn{6}{|l|}{ Antihypertensive treatment characteristics } \\
\hline \multicolumn{2}{|l|}{ Duration of therapy years (mean SD) } & $5.7(4.1)$ & $8.1(7.4)$ & ${ }^{*} 1.05$ (1.02 to 1.08$)$ & *1.05 (1.02 to 1.09$)$ \\
\hline \multirow[t]{2}{*}{ Adherence (MMAS $\geq 7)(n, \%)$} & No & $232(76)$ & $73(24)$ & Ref & \\
\hline & Yes & $178(78)$ & $49(22)$ & 0.88 (0.58 to 1.32$)$ & \\
\hline \multirow{2}{*}{$\begin{array}{l}\text { Therapy at prior visit } \\
(n, \%)\end{array}$} & Monotherapy & $168(76)$ & $53(24)$ & Ref & \\
\hline & Multidrug therapy & $249(78)$ & $70(22)$ & 0.89 (0.59 to 1.34$)$ & \\
\hline
\end{tabular}

Statistically significant values: ${ }^{*} p<0.05$. Variables with ${ }^{*} p<0.05$ or $+p<0.2$ in the bivariable model were included in the multivariable model. Variables with a $p<0.05$ in the multivariable model are indicated with * and set in bold typeface. Percentages are calculated per row. Treatment intensification was calculated for 540 patients who had complete medication history (including dose and frequency) on both visits and uncontrolled BP at current visit.

BP, blood pressure; MMAS, Morisky Medication Adherence Scale; MI, myocardial infarction; Ref, reference.

associated with a better BP control. Duration of therapy on antihypertensive medication was the only, although modestly, significant contributing factor of treatment intensification (also for achieving target BP).

When looking at other studies on hypertension awareness, treatment and control in Africa, 41 out of 44 studies showed a lower proportion of patients with controlled BP (these studies reported levels of control ranging from $<1 \%$ to $33 \%$ ) than our study. ${ }^{8}$ The reported wide variation could be explained by population differences and variation in study set-ups. The level of BP control in our study was between that reported in two studies performed in a Southern Ethiopian hospital. ${ }^{1623}$ Gudina et al studied the prevalence of hypertension among patients visiting a hospital for any reason, and of patients with known hypertension, $44 \%$ were controlled. ${ }^{23}$ In the 
other study, $50 \%$ of patients had achieved their target BP. ${ }^{16}$ This study was more of similar to ours; patients were included who visited an outpatient hypertension clinic and who had been treated for at least 12 months in the study hospital. ${ }^{16}$ Unfortunately, information on duration of the therapy was not included in these studies. ${ }^{16}{ }^{23}$ In comparison with studies from western countries, the percentages of patients with adequately controlled BP and those who received treatment intensification were lower in our study than in North American countries but similar to some European countries. ${ }^{24} 25$ These differences may be explained in part by different national guidelines recommendations. However, as reported elsewhere, it is not only differences between guidelines, but also how much effort countries put in implementation of these recommendations. ${ }^{25}$ While the Ethiopian guideline is similar to the USA guidelines, ${ }^{22}{ }^{24}$ possible differences in implementation, due to African factors including resource limitations, low priority for non-communicable diseases and healthcare providers' behaviour and skills may in part explain the low level of BP control. ${ }^{26}$ However, comparing our results with population-based studies in western countries or those in other parts of Africa should be done with caution as we investigated two regional Ethiopian hypertensive populations treated at a hospital setting only.

In our study, one of the determinants for achieving target $\mathrm{BP}$ was the healthcare setting. Patients who are referred to specialised hospitals may be more complex-in terms of comorbidities or severity of hypertension. Numerically, patients received more treatment intensification at specialised hospitals (27\%) than at generalised hospitals $(21 \%)$, although these differences were not significant in our bivariable and multivariable analyses (table 3 ). Thus, the additional effort provided in these specialised hospitals may have not been sufficient to offset the difficulties in achieving BP control in the more complex patient population. Younger age was another significant determinant for achieving target BP. Prescribers in our study may have accepted higher BP in older patients, possibly because of tolerability or perceived lack of need for tight BP control. Recent evidence, however, suggests that 'the lower is the better', also in older patients. ${ }^{27} 28$ Nevertheless, guidelines lack consistency on BP targets for the elderly, ${ }^{29}$ especially when patients are frail and doctors may not aim for tight BP control. Another determinant of BP control was the type of medication prescribed. Most of our study participants received diuretics, the firstline antihypertensive agents. We have no data in which order medication was initiated. Therefore, we can only speculate why treatment regimens containing these drugs did not show better BP control. Since three quarters of diuretics-containing regimens in our study existed of two drugs only (see online supplementary table 2), patients may need additional antihypertensive therapy.

Only one-fifth of patients with uncontrolled BP at the current visit had their treatment intensified. Longer treatment duration was the only statistically significant determinant for intensification. Possibly, it took some while before prescribers could intensify treatment. Ultimately, the lack of BP control at the prior visit was the strongest predictor of patients not having controlled BP at current visit. This seems to suggest some level of 'clinical inertia', where doctors are slow to respond to clinical parameters. This practice indicates a need to intensify therapy. Indeed, a lack of achieving BP control may also be explained by true therapy resistant hypertension (although only $17 \%$ of patients received three or more antihypertensive agents at the prior visit).$^{30-33}$ Moreover, prescribers may not intensify treatment if they suspect that increased BP levels may be related to a suspected or reported poor compliance for a particular patient. Poor medication adherence is known as an important determinant for controlling hypertension. ${ }^{34}$ The level of adherence we observed ( $40 \%$ and $57 \%$ for MMAS- 8 with a cut-off at $>6$ and $\geq 6$, respectively) was close to that reported by Asgedom et al (35\% and $61 \%$, respectively). ${ }^{16}$ Two other Ethiopian studies reported low levels of adherence, although more difficult to compare as they used a four-point MMAS. ${ }^{35}{ }^{36}$ Surprisingly, the level of adherence was not associated with BP control in our main and sensitivity analyses (see online supplementary table 1). Similarly in the study by Asgedom et al, a hospital-based study in Southern Ethiopia, no relation with adherence and BP control was observed. ${ }^{16}$ Self-reported medication adherence may be overestimated and therefore lead to bias.

We found that more women with hypertension than men were included in our study, and that few patients smoked. Our study was not a population study designed to evaluate prevalence of hypertension, and the reason why more women were included could have been that women seek more care than men. Although a recent community-based study evaluating prevalence of hypertension in Ethiopia suggested more women were hypertensive than men, ${ }^{33}$ a meta-analysis including hospital-based studies ${ }^{15}$ and another recent hospital-based study reported a higher prevalence of males with hypertension. ${ }^{16}$ The higher prevalence of women in our study does not appear to have a strong impact on our study findings, as gender was not a significant determinant for $\mathrm{BP}$ control $\mathrm{BP}$ or treatment intensification.

Poor hypertension control should be addressed in a holistic approach that includes lifestyle modification and management of comorbid illnesses. Our study was largely performed in urban areas with the highest prevalence of hypertension in Ethiopia, likely attributed to adoption of a Western lifestyle. ${ }^{15}$ Still, our patient population looks very different from that in European or USA studies, that is, few smokers and few patients with (known) cardiometabolic comorbidities.

\section{Strengths and limitations}

As far as we are aware, this was the first study of its kind in Ethiopia covering a relatively diverse population. Our data included patients from hypertension outpatient clinics of 
six public hospitals in the capital city and northern region of Ethiopia.

A limitation of our study was the validity of the BP measure used. We analysed BP measurements as recorded in patients' medical records that reflected actual clinical practice, but these values may be subject to recording and measurement error. It is not clear how prescribers considered measurement variability or if any attempt was made to avoid 'white-coat' hypertension, for example, by repeating BP measurement. Still, many observational studies use medical records-with data collected in routine practice-as a data source. Future studies may consider using standardised assessment of BP. In our study, the level of BP control was assessed for two consecutive visits only. Follow-up at more visits may still be needed, as achieving BP control may require more time and would thus provide a better understanding of doctors truly being slow to intensify treatment.

Another limitation is that medical records did not include extensive or well-structured patient information. For example, comorbidities may be under-reported. For this reason, we limited evaluated comorbidities to cardiometabolic diseases as these are relevant to hypertension prognosis and treatment and are more likely to have been recorded in the charts. We did not study if prescribing was in line with guideline recommendations, that is, based on comorbidities, but focused instead on the actual impact of prescribing on BP. This study focused on public secondary and specialised hospitals; therefore, the results may not be generalisable to other settings such as private practices and primary healthcare centres. Differences in socioeconomic status did not seem related with type of drug prescribed. This may have affected redeeming prescriptions at the pharmacy, but we did not have that information. We did not query patients for economic reasons of non-compliance, for example, if they could afford their medication or that they needed to travel too far to collect medication. We used the validated MMAS-8 questionnaire and did not want to overburden patients further. Nevertheless, educational status-a proxy for socio economic status-in our study population was not related to BP control.

Finally, as in all studies we were not able to include all previously reported potential confounders for achieving BP control. ${ }^{35}$ For example, type of prescriber (was difficult to retrieve from medication charts), or medication counselling and patient's own knowledge of hypertension and treatment goals (would have required further interview time) may require further study.

\section{CONCLUSION}

Nearly two-thirds of patients on antihypertensive medication did not achieve target BP during routine clinical follow-up, and only a quarter of these patients with uncontrolled $\mathrm{BP}$ received treatment intensification. To improve care for patients visiting Ethiopian hospital hypertension clinics, focus should be on older patients and interventions may be needed for specialised centres.

Acknowledgements The authors wish to thank the study participants, data collectors and study hospital administrators who contributed to this study. The authors thank Michelle Pena for her valuable comments to the paper.

Contributors DFB, KT and PGMM designed and performed the research, analysed and interpreted the data. FMH-R, AM, YTM designed the study. All authors participated in writing the manuscript, also read and approved the final version.

Funding The study was part of a PhD project to the first author funded by the Netherlands Organization for International Cooperation in Higher Education (NUFFIC).

Competing interests None declared.

Patient consent Obtained.

Ethics approval Ethiopian Health Research Ethical Review Committees of (1) the College of Health Sciences, Mekelle University, (2) St Paul's Hospital Millennium Medical College and (3) the Department of Internal Medicine, School of Medicine, College of Health Sciences, Addis Ababa University.

Provenance and peer review Not commissioned; externally peer reviewed. Data sharing statement № additional data are available for this specific study. Open Access This is an Open Access article distributed in accordance with the Creative Commons Attribution Non Commercial (CC BY-NC 4.0) license, which permits others to distribute, remix, adapt, build upon this work non-commercially, and license their derivative works on different terms, provided the original work is properly cited and the use is non-commercial. See: http://creativecommons.org/ licenses/by-nc/4.0/

(c) Article author(s) (or their employer(s) unless otherwise stated in the text of the article) 2017. All rights reserved. No commercial use is permitted unless otherwise expressly granted.

\section{REFERENCES}

1. World Health Organization. A global brief on hypertension: silent killer: Global public health crisis, 2013. http://apps.who.int/iris/ bitstream/10665/79059/1/WHO_DCO_WHD_2013.2_eng.pdf. (accessed 17 May 2016).

2. Fuchs FD. Why do black Americans have higher prevalence of hypertension?: an enigma still unsolved. Hypertension 2011;57:379-80.

3. Brewster LM, Seedat YK. Why do hypertensive patients of African ancestry respond better to calcium blockers and diuretics than to ACE inhibitors and $\beta$-adrenergic blockers? A systematic review. BMC Med 2013;11:11.

4. Opie LH, Seedat YK. Hypertension in sub-Saharan African populations. Circulation 2005;112:3562-8.

5. Sagnella GA. Why is plasma renin activity lower in populations of African origin? J Hum Hypertens 2001;15:17-25.

6. Dennison CR, Peer N, Steyn K, et al. Determinants of hypertension care and control among peri-urban Black South Africans: the $\mathrm{HiHi}$ study. Ethn Dis 2007;17:484-91.

7. James PA, Oparil S, Carter BL, et al. evidence-based guideline for the management of high blood pressure in adults: report from the panel members appointed to the Eighth Joint National Committee (JNC 8). JAMA 2014;2014:507-20.

8. Kayima J, Wanyenze RK, Katamba A, et al. Hypertension awareness, treatment and control in Africa: a systematic review. BMC Cardiovasc Disord 2013;13:54.

9. Kessler CS, Joudeh Y. Evaluation and treatment of severe asymptomatic hypertension. Am Fam Physician 2010;81:470-6.

10. van de Vijver S, Akinyi $\mathrm{H}$, Oti S, et al. Status report on hypertension in Africa-consultative review for the 6th Session of the African Union Conference of Ministers of Health on NCD's. Pan Afr Med J 2013;16:38.

11. Naicker S, Plange-Rhule J, Tutt RC, et al. Shortage of healthcare workers in developing countries-Africa. Ethn Dis 2009;19(Suppl 1):S160-4.

12. Mancia G, Fagard R, Narkiewicz K, et al. ESH/ESC guidelines for the management of arterial hypertension: the task force for the management of arterial hypertension of the European Society of 
Hypertension (ESH) and of the European Society of Cardiology (ESC). Blood Press 2013;2013:193-278.

13. Abebe SM, Berhane $Y$, Worku A, et al. Prevalence and associated factors of hypertension: a crossectional community based study in Northwest Ethiopia. PLoS One 2015;10:e0125210.

14. Adeloye D, Basquill C. Estimating the prevalence and awareness rates of hypertension in Africa: a systematic analysis. PLoS One 2014;9:e104300.

15. Kibret KT, Mesfin YM. Prevalence of hypertension in Ethiopia: a systematic meta-analysis. Public Health Rev 2015;36:14.

16. Asgedom SW, Gudina EK, Desse TA. Assessment of Blood pressure control among hypertensive patients in Southwest Ethiopia. PLoS One 2016;11:e0166432.

17. Shukrala F, Gabriel T. Assessment of prescribing, dispensing, and patient use pattern of antihypertensive drugs for patients attending outpatient department of Hiwot Fana Specialized University Hospital, Harar, Eastern Ethiopia. Drug Des Devel Ther 2015;9:519-23.

18. Federal Democratic Republic of Ethiopia Ministry of Health. Health sector transformation plan (HSTP)-2015/16 - 2019/20: Ethiopia Ministry of Health, 2015:142. http://www.moh.gov.et/documents/ 26765/0/Health+Sector+Transformation+Plan/5542a23a-9bc7-46a28c1f-8b32c2603208?version=1.0 (accessed 3 Mar 2017).

19. Lee GK, Wang HH, Liu KQ, et al. Determinants of medication adherence to antihypertensive medications among a chinese population using Morisky Medication adherence Scale. PLoS One 2013;8:e62775

20. Morisky DE, Ang A, Krousel-Wood M, et al. Predictive validity of a medication adherence measure in an Outpatient setting. $J$ Clin Hypertens 2008;10:348-54.

21. Beaton DE, Bombardier C, Guillemin F, et al. Guidelines for the process of cross-cultural adaptation of self-report measures. Spine 2000;25:3186-91.

22. FMHACA. Hypertension: Standard Treatment Guidelines for General Hospitals, Ethiopia. Third Edition: Food, Medicine and Health Care Administration and Control Authority (FMHACA), 2014:47-53.

23. Gudina EK, Michael Y, Assegid S. Prevalence of hypertension and its risk factors in southwest Ethiopia: a hospital-based cross-sectional survey. Integr Blood Press Control 2013;6:111-7.
24. Wang YR, Alexander GC, Stafford RS. Outpatient hypertension treatment, treatment intensification, and control in Western Europe and the United States. Arch Intern Med 2007;167:141-7.

25. Wolf-Maier K, Cooper RS, Kramer H, et al. Hypertension treatment and control in five European countries, Canada, and the United States. Hypertension 2004;43:10-17.

26. Nulu S, Aronow WS, Frishman WH. Hypertension in Sub-Saharan Africa: a contextual view of patterns of disease, best management, and systems issues. Cardiol Rev 2016;24:30-40.

27. Wright JT, Williamson JD, Whelton PK, et al. A randomized trial of intensive versus standard blood-pressure control. N Engl J Med 2015;373:2103-16.

28. Perkovic V, Rodgers A. Redefining blood-pressure targets-SPRINT starts the marathon. N Engl J Med 2015;373:2175-8.

29. Alhawassi TM, Krass I, Pont LG. Hypertension in older persons: a systematic review of National and International Treatment guidelines. J Clin Hypertens 2015;17:486-92.

30. Phillips LS, Branch WT, Cook CB, et al. Clinical inertia. Ann Intern Med 2001;135:825-34.

31. Moser M. Physician or clinical inertia: what is it? Is it really a problem? And what can be done about it? J Clin Hypertens 2009;11:1-4.

32. Achelrod D, Wenzel U, Frey S. Systematic review and meta-analysis of the prevalence of resistant hypertension in treated hypertensive populations. Am J Hypertens 2015;28:355-61.

33. Nansseu JR, Noubiap JJ, Mengnjo MK, et al. The highly neglected burden of resistant hypertension in Africa: a systematic review and meta-analysis. BMJ Open 2016;6:e011452.

34. Corrao G, Parodi A, Nicotra F, et al. Better compliance to antihypertensive medications reduces cardiovascular risk. $J$ Hypertens 2011;29:610-8.

35. Ambaw AD, Alemie GA, W/Yohannes SM, et al. Adherence to antihypertensive treatment and associated factors among patients on follow up at University of Gondar Hospital, Northwest Ethiopia. BMC Public Health 2012;12:282.

36. Hareri HA, Abebe M, Asefaw T. Assessments of adherence to hypertension managements and its influencing factors among hypertensive patients attending black lion hospital chronic follow up unit, Addis Ababa, Ethiopia-a cross-sectional study. Int J Pharm Sci Res 2013;4:1086. 